\title{
Influence of thermo hydrogen treatment on microstructure and mechanical properties of Ti-5Al-2.5Sn ELI alloy
}

\author{
*Ya-fei Ren ${ }^{1,2}$, Jun Zhao ${ }^{1,2}$, Hua-sheng Xie ${ }^{1,3}$, Shi-bing Liu ${ }^{1,2}$, Yan-chun Lou ${ }^{1,2}$, and Ping Yan ${ }^{1,2}$ \\ 1. Shenyang Research Institute of Foundry, Shenyang 110022, China; \\ 2. State Key Laboratory of Light Alloy Casting Technology for High-end Equipment, Shenyang 110022, China; \\ 3. Beijing Research Institute of Mechanical \& Electrical Technology, Beijing 100083, China
}

\begin{abstract}
Thermo hydrogen treatment (THT) of titanium is a process in which hydrogen is used as a temporary alloying element in titanium alloys. It is an attractive approach for controlling the microstructure and thereby improving the final mechanical properties. In the present study, the microstructure of the original (non-hydrogenated) sample has only a phase and the grains is coarse with an average size of $\sim 650 \mu \mathrm{m}$. While the grain size of thermo hydrogen treated Ti-5AI-2.5Sn ELI alloy became finer and the mechanical properties were improved significantly. When the hydrogen content of the hydrogenated Ti-5Al-2.5Sn ELI alloy is $0.321 \mathrm{wt} . \%$, $\beta$ phase and $\delta$ titanium hydride appear. Also the average grain size decreases to $450 \mu \mathrm{m}$. When the hydrogen content is $0.515 \mathrm{wt} . \%$, the grain size decreases to $220 \mu \mathrm{m}$. The mechanical properties were tested after dehydrogenation, and the mechanical properties improved significantly compared to the unhydrogenated specimens. The tensile strength of the Ti-5Al$2.5 \mathrm{Sn}$ ELI alloy improved by $17.7 \%$ when the hydrogen content increased to $0.920 \mathrm{wt} . \%$, at the same time the percentage reduction of area $(Z)$ increased by $33 \%$ and the impact toughness increased by $37 \%$.
\end{abstract}

Key words: thermo hydrogen treatment; microstructure; mechanical properties

CLC numbers: TG146.23 Document code: A Article ID: 1672-6421(2017)01-055-04

$\mathrm{T}$ itanium alloys are widely used in various fields because of their characteristics of low density, high strength, corrosion resistance and high temperature resistance. Ti-5Al-2.5Sn ELI alloy is a kind of important structural material used under low temperature in the aerospace field. To meet the need of Ti-5 Al-2.5Sn ELI alloy castings with high strength and high fatigue resistance, this work intends to research the influence of thermo hydrogen treatment (THT) on this alloy.

The thermo hydrogen process of titanium alloys can change the microstructure and improve the mechanical properties of the alloys due to hydrogen-refined grains, hydrogen-induced phase transformations and reversible reaction of hydrogen with titanium by using hydrogen as a temporary alloying element ${ }^{[1]}$.

Hydrogen is a unique alloying element in titanium alloys, because it can be easily added and removed without

\section{*Ya-fei Ren}

Male, born in 1989, Master's degree. Research direction: casting technologies of titanium alloys.

*Corresponding author: louych@chinasrif.com

Received: 2016-10-21; Accepted: 2016-12-20 melting the alloys. Titanium and its alloys have a high affinity with hydrogen, being capable of absorbing up to 60at. $\%$ hydrogen at $600{ }^{\circ} \mathrm{C}$, and even higher contents can be alloyed with titanium at higher temperatures ${ }^{[2]}$. Since the beginning of the titanium industry in the late 1940s, a lot of attention has been given to control the hydrogen content of titanium products as hydrogen levels above $0.02 \mathrm{ppm}$ can lead to degradation in fracture-related mechanical properties ${ }^{[2-4]}$. Fortunately, the reaction of titanium and hydrogen is reversible due to a positive enthalpy of solution in titanium ${ }^{[5,6]}$, allowing hydrogen to be easily removed by vacuum annealing.

Cast Ti-5Al-2.5Sn ELI alloy has low mechanical properties due to the formation of porosity and unfavorable microstructure (coarse grains and large-flake intragrain structure). The porosity can be removed partially or fully by hot isostatic pressing (HIP). The conventional heat treatment, i.e., annealing aimed at removing the residual casting stresses, does not increase the strength of Ti-5Al-2.5Sn ELI alloy. THT based on reversible alloying with hydrogen is a novel and effective method for refining the structure of titanium alloys and thereby improving their mechanical properties. The present work was aimed at enhancing the mechanical properties of cast Ti-5Al-2.5Sn ELI alloy by THT. 


\section{Experimental procedure}

The materials used in this experiment were as-cast Ti-5Al2.5Sn ELI alloy bars with $15 \mathrm{~mm}$ diameter. It was melted in a vacuum skull furnace of consumable electrode. The electrode was alloy ingot of Ti-5Al-2.5Sn ELI. And the mould was made of graphite. After cast, the bars were treated by hot isostatic pressing. Its chemical composition is shown in Table 1.

Table 1: Chemical compositions of Ti-5Al-2.5Sn ELI alloy (wt.\%)

\begin{tabular}{ccccccccc} 
Al & Sn & Fe & Si & C & N & H & O & Ti \\
\hline 5.33 & 2.36 & 0.097 & 0.069 & 0.015 & 0.011 & 0.0031 & 0.053 & Bal.
\end{tabular}

The specimens for hydrogenation treatment were all taken from the received bars poured in the same melting batch, and polished with $800 \#$ and 1000\# sandpapers, then cleaned ultrasonically in acetone solution, and finally dried in air.

Thermo hydrogen treatment for the Ti-5Al-2.5Sn ELI alloy consists of the following four processes: 1) The specimens are first hydrogenated in an atmosphere of hydrogen in a quartz tube-type furnace at $750{ }^{\circ} \mathrm{C}$ for $90 \mathrm{~min}$. The actual hydrogen contents in specimens are controlled by changing the equilibrium pressure of hydrogen; 2) Furnace cooled to room temperature; 3) Dehydrogenate the hydrogenated specimens in vacuum environment of $1 \times 10^{-3} \mathrm{~Pa}$ in a stainless steel tube-type furnace at $750{ }^{\circ} \mathrm{C}$ for $12 \mathrm{~h}$; 4) Furnace cooled to room temperature. The actual hydrogen content absorbed in specimens was measured by weighing the specimens before and after hydrogenation treatment using a high precision electronic balance (XPE204S) with the mass readability of $0.01 \mathrm{mg}$. In order to exclude the effect of heat treatment during hydrogenation on the properties of Ti-5Al-2.5Sn ELI alloy, the as-received Ti-5Al-2.5Sn ELI alloy was also produced by the same heat treatment except the hydrogenatation.

To observe the microstructure, the etching solution of $\mathrm{HF}(10 \mathrm{~mL})+\mathrm{HNO}_{3}(30 \mathrm{~mL})+\mathrm{H}_{2} \mathrm{O}(60 \mathrm{~mL})$ was used to etch the dehydrogenated specimens. And the microstructure of Ti-5Al-2.5Sn ELI was identified by an OLYCIA m3 optical microscope observation and a transmission electron microscope (JEOL JEM-2100F). The grain size for each specimen was measured by the linear interception method. Phase composition was analyzed by X-ray diffractometry (XRD, RIGAKU D/ MAX2500V) with $\mathrm{Cu} \mathrm{K} \alpha$ radiation under $100 \mathrm{kV}$ and $40 \mathrm{~mA}$, scanning parameter of $3^{\circ} \cdot \mathrm{min}^{-1}$. The tensile test was conducted on an electronic universal testing machine of CSS-1120 at room temperature with a strain rate of $5.556 \times 10^{-4} \mathrm{~s}^{-1}$. The impact test was conducted using an impact testing machine of JB-30B.

\section{Results}

\subsection{Microstructure transformation of Ti-5Al- 2.5Sn ELI after THT}

From Fig.1(a), it can be seen that the microstructure of the original sample only consists of $\alpha$ phase and the grains is coarse.

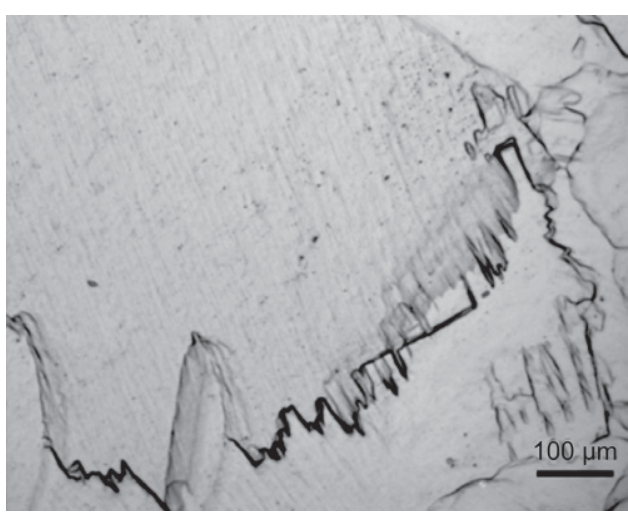

(a) Original sample

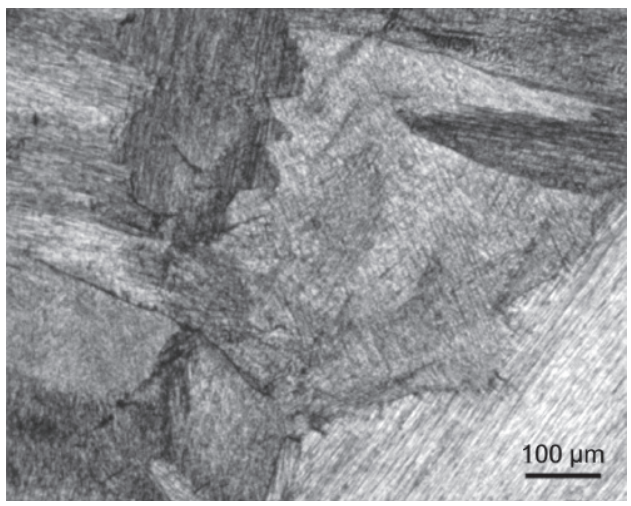

(c) $0.321 \mathrm{wt} . \% \mathrm{H}$

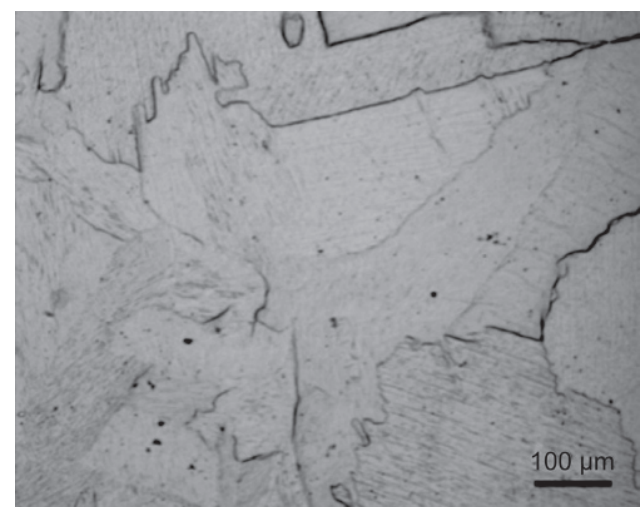

(b) $0.105 w t . \% \mathrm{H}$

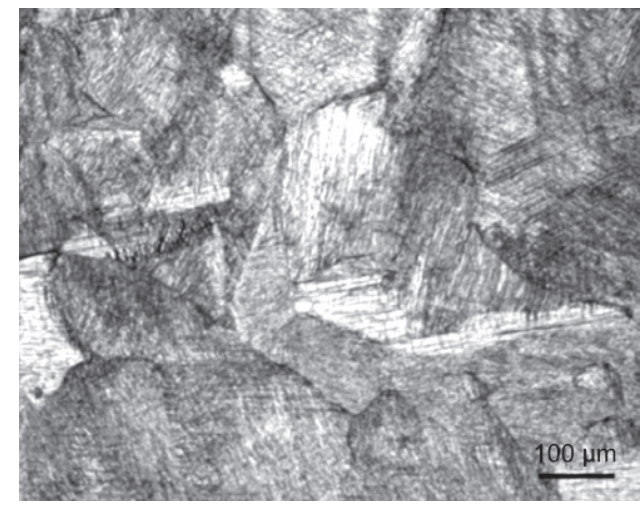

(d) $0.515 w t . \% \mathrm{H}$

Fig. 1: Influence of hydrogen concentration on microstructures of Ti-5AI-2.5Sn ELI alloy 
The average size is $650 \mu \mathrm{m}$. When the hydrogen concentration is $0.105 \mathrm{wt} . \%$, there is no obvious change of the microstructure and hydrogen atoms exist in the alloy mainly in the form of solid solution and no hydride appears. The average size becomes $450 \mu \mathrm{m}$ when the hydrogen concentration reaches $0.321 \mathrm{wt} . \%$. And when the hydrogen concentration increases to $0.515 \mathrm{wt} . \%$, the average size of grains decreases to $220 \mu \mathrm{m}$. So, little hydrogen has less influence on the microstructure of the alloy. With the increase of hydrogen concentration, the change of the microstructure becomes more obvious.

\subsection{XRD analysis of Ti-5Al-2.5Sn ELI after THT}

XRD patterns of the alloy with different hydrogen concentrations from $0.105 \mathrm{wt} . \%$ to $0.920 \mathrm{wt} . \%$ are shown in Fig. 2. It can be seen that the pattern of Ti-

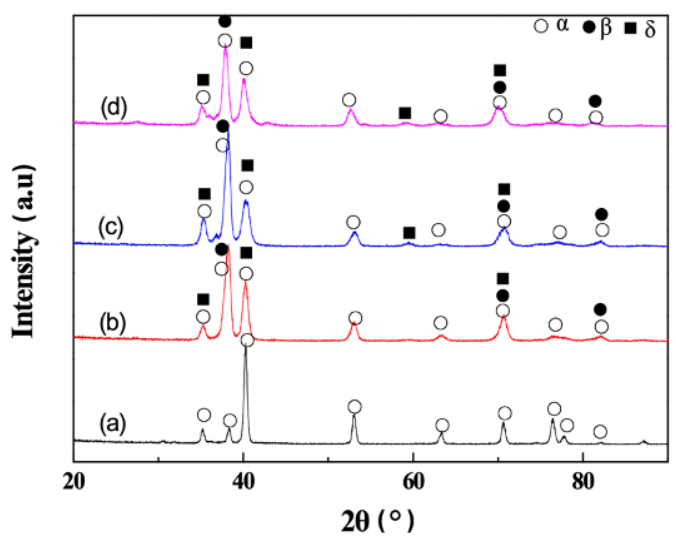

Fig. 2: XRD patterns of hydrogenated Ti-5AI2.5Sn ELI alloy: (a) $0.105 w t . \% \mathrm{H}$;

(b) $0.321 w t . \% \mathrm{H}$; (c) $0.515 w t . \% \mathrm{H}$; (d) 0.920 wt. $\% \mathrm{H}$
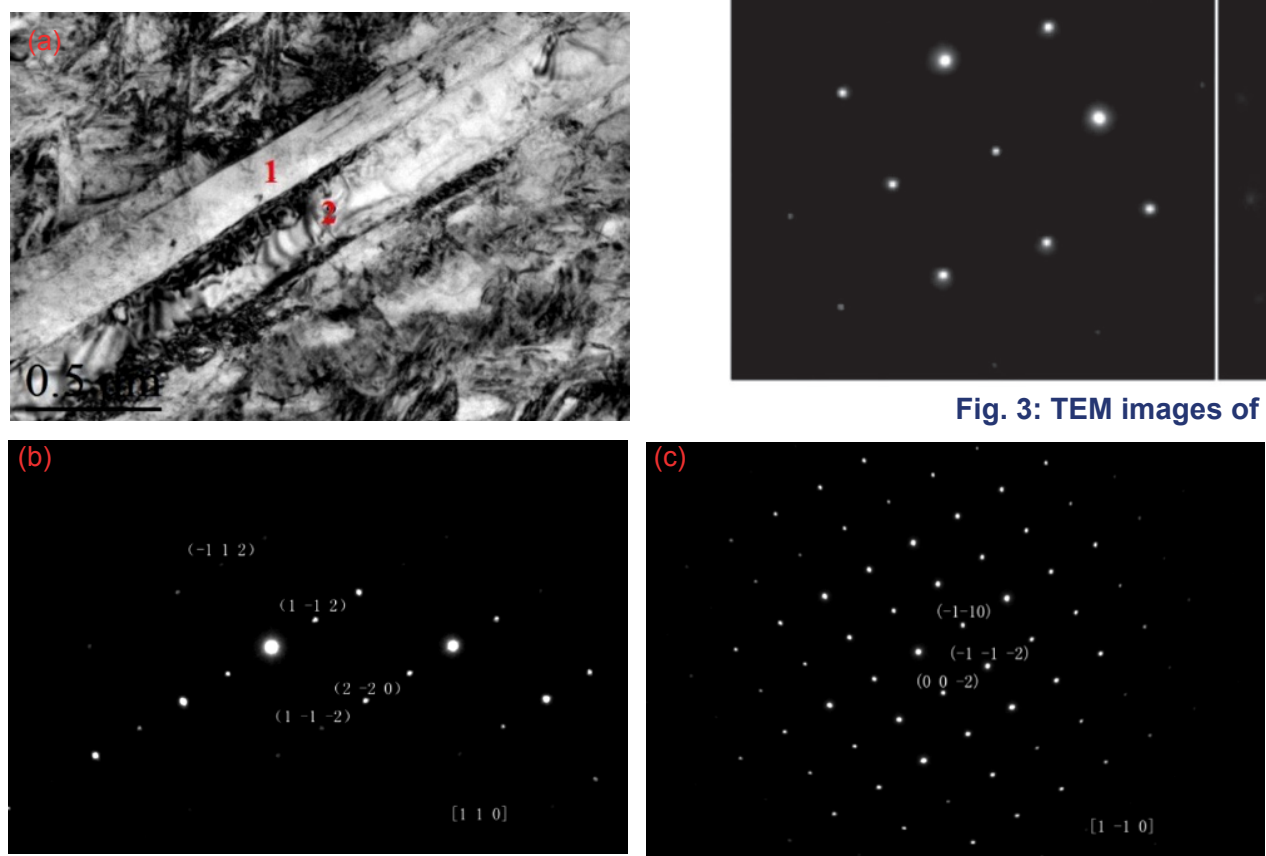

Fig. 4: TEM images of Ti-5AI-2.5Sn ELI alloy with $0.515 w t . \%$ hydrogen
5Al-2.5Sn ELI alloy with $0.105 w t . \%$ is the same as that of the original and only made of $\alpha$ phase. When the hydrogen concentration is higher, there are $\beta$ and $\delta$ phases to appear in the alloy.

The THT temperature in this experiment is $750{ }^{\circ} \mathrm{C}$ which is lower than the $\beta$ phase-transition temperature $1,020^{\circ} \mathrm{C}$ of Ti-5Al-2.5Sn ELI alloy. So hydrogen in the alloy can lower the $\beta$ phase-transition temperature ${ }^{[7]}$. Also the phase transition of $\alpha \rightarrow \beta$ and the transition of $\alpha_{H} \rightarrow \alpha \rightarrow \delta$ happen $^{8]}$.

\subsection{Hydride in Ti-5Al-2.5Sn ELI alloy after hydrogenating}

Transmission electron microscopy (TEM) study is performed to show that the hydride emerges in the alloy after THT. The results are shown in Figs. 3 and Fig. 4.

Figure 3 shows that there is only $\alpha$ phase, no $\delta$ phase in the original sample. While in the alloy with $0.515 \mathrm{wt} . \%$ hydrogen, lots of lath-like structures emerge (Fig. 4). By analysis of selected area of electron diffraction, the lath-like structures are shown to be $\delta$-phase hydrides. And the average width of the structures is only $0.2 \mu \mathrm{m}$, so it can not be seen under optical microscopy.

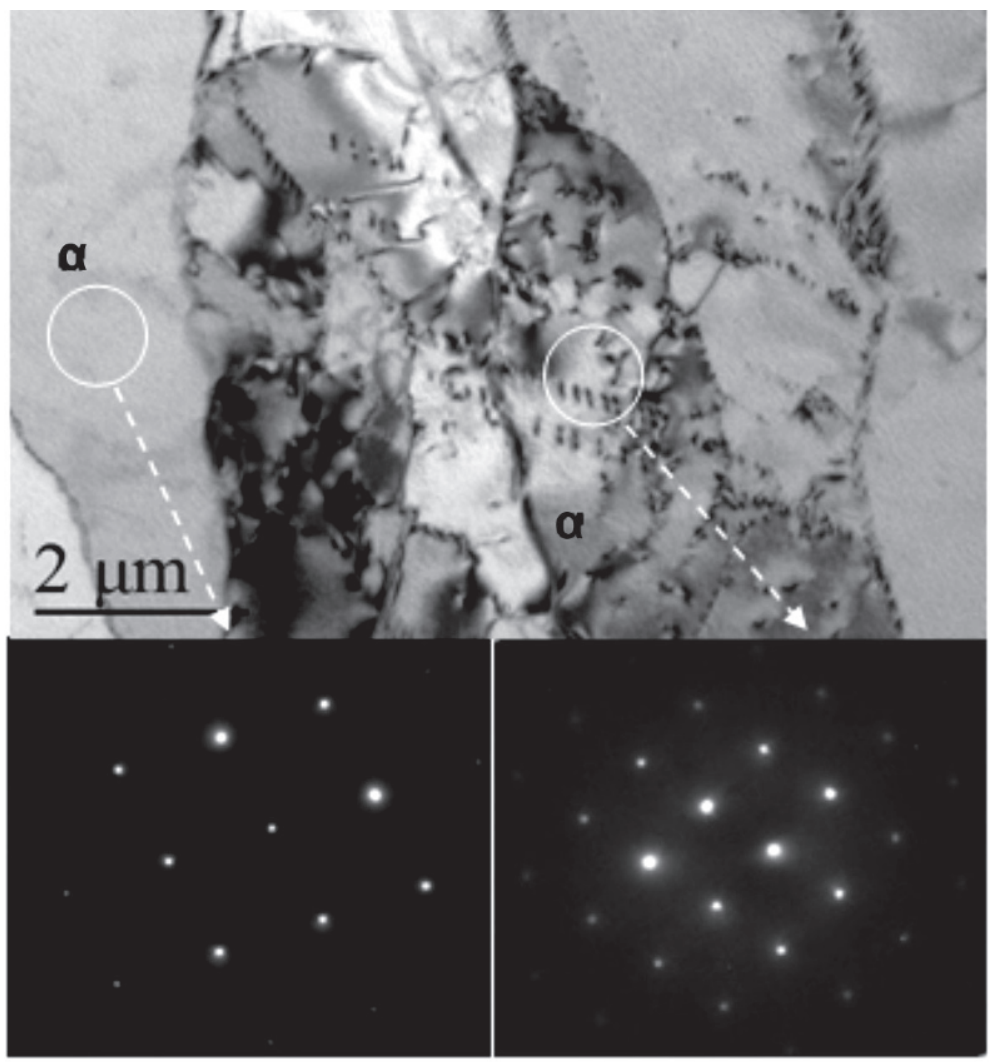

Fig. 3: TEM images of original Ti-5Al-2.5Sn ELI alloy 


\subsection{Influence of THT on mechanical properties}

Figures 5 and 6 show the mechanical properties of the dehydrogenated alloy samples. It can be seen from Fig. 5 that the tensile properties of the original sample do not change obviously after heat treatment. The ordinate axis of Fig. 5(a) shows the ductility of the alloy. The ductility reduced a little when the hydrogen content is $0.105 w t . \%$. Then the ductility becomes better with the increase of hydrogen content. When the hydrogen content is $0.920 \mathrm{wt} . \%$, the percentage reduction of area $(Z)$ increased by $33 \%$. At the same time the tensile strength improved by $17.7 \%$ and the impact toughness increased by $37 \%$ (Fig. 6 ).

\section{Discussion}

Due to the function of hydrogen, when the temperature is higher than the $\beta$ transformation temperature, the $\alpha$ grains will transform into $\beta$ phase ${ }^{[9]}$. Hydrogen can even lower the phase transformation point of titanium alloy. The $\alpha / \beta$ transformation temperature of Ti-5Al-2.5Sn ELI alloy is $1,020{ }^{\circ} \mathrm{C}{ }^{[10]}$. When hydrogenated at the temperature of $750{ }^{\circ} \mathrm{C}$ some of the $\alpha$ phase in Ti-5Al-2.5Sn ELI alloy has a transformation of $\alpha \rightarrow \beta$. So the phase of Ti-5Al-2.5Sn ELI alloy turns into $\alpha+\beta$. And with the increase of hydrogen, $\alpha$ and $\beta$ phases in the alloy then become $\alpha_{\mathrm{H}}$ and $\beta_{\mathrm{H}}$. In the process of furnace cooling, the transformations of $\beta_{H} \rightarrow \alpha+\delta$ (hydride) and $\alpha_{H} \rightarrow \alpha+\delta$ appear. And there will be little $\beta$ phase left in the alloy. During the dehydrogenation, reaction of $\delta \rightarrow \alpha+\mathrm{H}_{2}$ happens. So the hydride decomposes to $\alpha$ phase. And the new $\alpha$ phase takes the position of the $\delta$ phase. The structure will become "lamellar $\alpha /$ fine $\beta /$ lamellar $\alpha$ ", so the grains is finer than the original $\alpha$ phase.

The grain size has an important influence on the mechanical properties of titanium alloy. With the increase of hydrogen, the grains become finer, so the mechanical properties of Ti-5Al2.5Sn ELI alloy is improved.

\section{Conclusions}

(1) After hydrogenation and dehydrogenation treatment, there will be $\delta$ hydride emerging in the Ti-5Al-2.5Sn ELI alloy which has an influence on the microstructure. When the hydrogen content of Ti-5Al-2.5Sn ELI is 0.321 wt. $\%, \beta$ phase and $\delta$ titanium hydride are found in Ti-5Al-2.5Sn ELI. The average grain size decreases to $450 \mu \mathrm{m}$ from the original size of 650 $\mu \mathrm{m}$. When the hydrogen content is $0.515 \mathrm{wt} . \%$, the grain size decreases to $220 \mu \mathrm{m}$. The grain refinement is controlled by phase transformation.

(2) The THT improves the mechanical properties significantly. The tensile strength is improved by $17.7 \%$ when the hydrogen

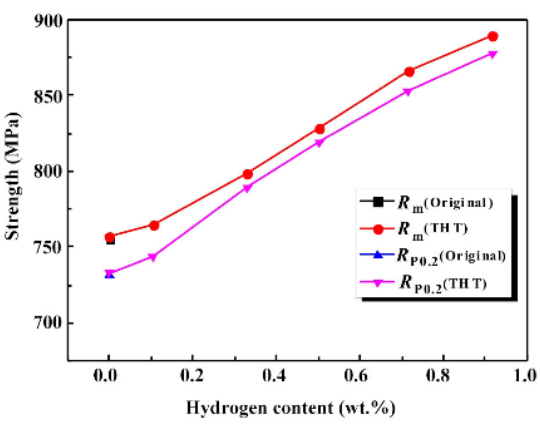

ensile properties of Ti-5AI-2.5Sn ELI alloy as a function of hydrogen concentration

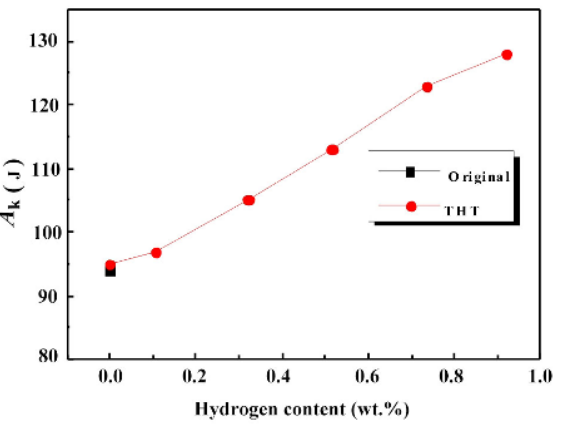

Fig. 6: Impact energy of Ti-5Al-2.5Sn ELI alloy as a function of hydrogen concentration

content is $0.920 \mathrm{wt} . \%$, at the same time the percentage reduction of area $(Z)$ increases by $33 \%$ mean while the impact toughness increases by $37 \%$.

\section{References}

[1] Smickley R J, Sardi E. US Patent 4505764, March 19, 1985.

[2] San-Martin, Manchester F D. The H-Ti (Hydrogen-Titanium) System. Bulletin of Alloy Phase Diagrams, 1987, 8(1): 30-42.

[3] Lenning G A, Graighead C M, Jaffee R I. Constitution and Mechanical Properties of Titanium-Hydrogen Alloys. Trans. AIME, 1954, 200: 367-76.

[4] Völkl J, Alefeld G. Diffusion of hydrogen in metals. Hydrogen in Metals III. Wipf H, editor, Springer Berlin Heidelberg, 1997, 73(3): 246.

[5] McQuillan A D. An Experimental and Thermodynamic Investigation of the Hydrogen-Titanium System, Proc. Royal Society of London, Series A 1951, 204: 309-22.

[6] Senkov O N, Jonas J J, Froes F H. Recent Advances in the Thermohydrogen Processing of Titanium Alloys. Over-view. JOM, 1996;48(7): 42-7.

[7] Martin S, Manchester F D. The H-Ti (Hydrogen-Titanium) System. Bulletin of Alloy Phase Diagrams, 1987, 8(1): 30-42.

[8] Kohn D H, Ducheyne P. Tensile and fatigue strength of hydrogen-treated Ti-6Al-4V alloy. Journal of Materials Science, 1991, 26(2): 328-334.

[9] Fang $\mathrm{T} Y$, Wang $\mathrm{W} \mathrm{H}$. Microstructural features of thermochemical processing in Ti-6Al-4V alloy. Materials Chemistry and Physics, 1998, 56: 35-47.

[10] Shi Kun. Study on Microstructures and Mechanical Properties of Ultralow Temperature Ti-5Al-2.5Sn ELI Cast Alloy. Dissertation, Shenyang Research Institute of Foundry, 2008: 34-37. (In Chinese) 